\title{
Developing a Near-optimal Lowest-consumption Tunnel Lighting System Using Software Agents through Power Line Communications
}

\author{
Nicolaos Protogeros ${ }^{1}$, Anastasios A. Economides ${ }^{1}$, Grigoris K. Papagiannis ${ }^{2}$ \\ and Chris Syleos ${ }^{2}$ \\ ${ }^{1}$ University of Macedonia, Thessaloniki, Greece \\ ${ }^{2}$ Aristotle University of Thessaloniki, Greece
}

\begin{abstract}
This paper describes an innovative Energy Consumption Management System (ECMS) that is aimed at rationalizing the end use of the electrical energy in tunnel lighting. It is based on agent technologies and Power Line Communications to achieve lowest consumption lighting conditions in an optimal way. Depending on the type of application, the system would lead also to reduced installation and running costs, especially as far as lighting systems are concerned.
\end{abstract}

Keywords: energy consumption management, lowest consumption, power line communications, software agents, street lighting, tunnel lighting

\section{Introduction}

Multi-agent solutions represent one of the most promising technological paradigms, especially when applied to complex and highly parallel environments where the problem is too difficult to be modeled and solved with traditional monolithic applications.

Many organizations are working towards the standardization and realization of development environments and frameworks to support the development of agent-oriented systems. These platforms allow the development of simple software that models complex behaviors. The tunnel lighting problem is a complex one if we want to apply lowest consumption techniques. The application of software agents has challenged us to try and resolve the problem of optimal tunnel and street lighting with lowest consumption in the power domain.
The proposed system is based on the Energy Consumption Management System (ECMS) of PowerManSwitch (PMS) Engineering (Nilsson, 2003). It is a system implemented as a set of devices that can be used in street lighting installations to control the lamps. The punctual control devices are driven using Power Line Communication (Tsiamitros, 2003). The system was realized within the frame of a 5th Framework European Research Project (PMS). During the development of this project traditional software and protocols were implemented to control on/off switching and regulate such devices. However, since traditional software solutions do not offer characteristics like collaboration among the parts of the lighting system in order to achieve the optimal solution and also autonomy for each part to act independently, we decided to attempt a new approach for the software by applying multi-agent technologies to this domain.

In this paper, we present the architectural approach along with the results achieved from the execution of a system's prototype developed with JADE (JADE), where agents compete in order to minimize power consumption. JADE was chosen as the agent framework, since it is one of the available frameworks that can be deployed on small devices and facilitates the development of agent applications (Krzysztof, 2005).

The rest of the paper is organized as follows. Section 2 describes relevant agent applications. Section 3 presents the street lighting problem 
and the initial solution developed using traditional software paradigms. Section 4 describes the tunnel lighting problem and its challenges. Then the JADE agents are applied to this specific problem domain. Finally, Section 5 concludes with the experimental results and with on-going developments.

\section{Related Research}

In recent years many studies have been carried out concerning the application of software agents to the power industry. Agents have been used in developing markets where power trading takes place, for power load balancing, and for energy management. However, there in no application of software agents to the tunnel lighting problem, as far as we know. In the following, we examine some of the applications of software agents to the power domain. The only similarity with these approaches is the application of software agents and the field of application is the power domain.

One such application of software agents to computational markets was done by Ygge et al. (1996). Computational markets are based on computational agents (Ygge and Akkermans, 1996). Computational markets have been suggested as a solution to complex resource allocation, scheduling, and optimization problems (Kurose and Simha, 1989; Lenting and Braspenning, 1993; Gagliano et al., 1995). Ygge et al. (1996) sketch how direct load management can be modeled as a computational market.

Software agents were also utilized for direct power load management (Ygge, 1998). These agents were extended for direct trade in power pools (Ygge, 1999). An agent for energy management at the customer side, which was called a HomeBot, used the customer preferences (e.g. how much the customer is prepared to pay for maintaining a certain indoor temperature), load model (e.g. how much energy it takes to heat a room to a certain temperature, and how much energy is dissipated to the environment through walls and windows), disturbance predictions (e.g. if people will be present during the period under consideration and what the weather will be like), and price predictions, to compute a utility function. The utility function expressed the valuation of the resource (i.e. electrical power).
Another application of software agents concerned load balancing of the energy demand. This application has a large industrial value, since the utility can avoid costly and sometimes environment-unfriendly generation of energy to meet peak demands. Furthermore, investments in expensive power generation and distribution equipment to take care of peak demand can thus also be trimmed. A win-win situation for utilities and customers can be obtained by trading energy allocation versus price or customer value, thus agreeing on a suitable redistribution profit for all parties. Gustavsson (1999) modeled the load-balancing task as a multi-agent system and introduced a computational market with auctions as an allocation mechanism (Akkermans and Ygge, 1997). The allocation of agents follows the topology of the electric grid. Device agents, called HomeBots, intermediate Service agents, and the Utility agent were the participants in the auction. The natural hierarchy of agents induced by the network topology allowed the design and implementation of highly efficient distributed auction algorithms. A simulation of the multi-agent system illustrated that after a change in price by the utility agents, total demand and redistribution of energy was done in just a few steps (Ygge, 1996; Ygge, 1998; Akkermans, 1996).

\section{Street Lighting and Devices}

ECMS has two entities of main players: a) the user, and b) the loads to be managed. Therefore, ECMS needs user terminal equipment (UTE) and smart load terminals called, in our case, Power Management Switches (PMS). As the geographical distance between the UTE and PMS is increasing, the system can be considered as Wide Area Network (WAN). Network system management and security issues led to the design shown in Figure 1, which includes UTE, $\mathrm{PMS}$, and the necessary equipment to complete the system. The loads will be controlled by their PMS which belong to a secondary power transformer, and constitute a LAN. PMS units within this LAN communicate via PLC with a unit called Command Unit (CMU). Depending on PLC transmission capabilities, more than one CMU may be needed. Each CMU has within its transmission range area many PMS units. CMU is used for rooting requests from the outside region to the proper PMS and vice versa. It has also built-in intelligence to be used as a server for the requirements of the system concerning 


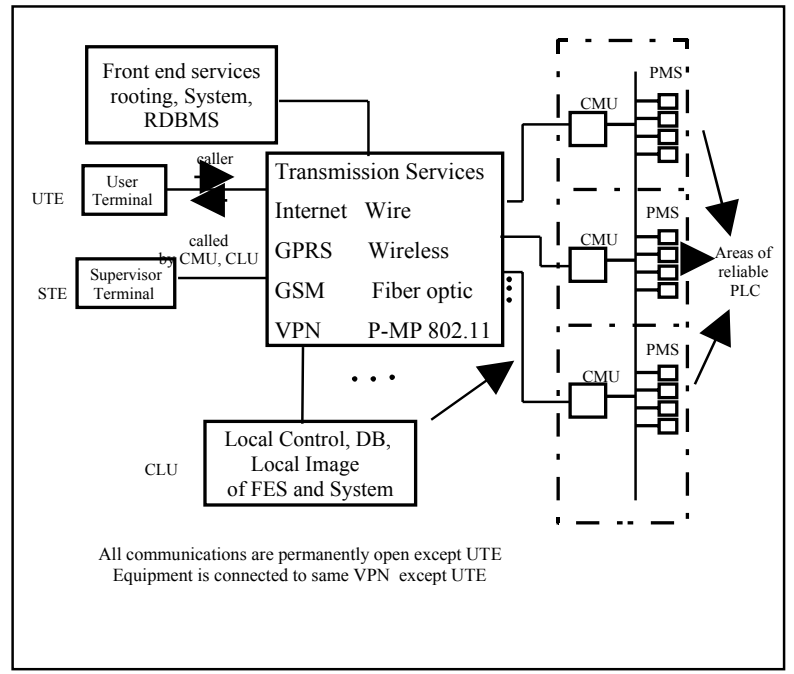

Figure 1. Energy Control Management System (ECMS).

the LAN attached to it and the connection to the outside network. CMUs belonging to different secondary power transformers are connected to a Control Unit (CLU), which can be accessed by the user either directly or remotely via other commercially available services. For the connection between CLU and CMU, all available public services, such as GPRS or GSM telephony, can be used. Direct fiber optic, copper links or wireless Ethernet protocol 802.11b connections are also applicable. CLU has all the intelligence needed to control and serve the LAN attached to it.

CLU keeps historical data, it offers optimization platforms for optimal control, evaluates messages, produces reports, etc.

For more extended systems covering a large geographical area, the use of public transmission services are needed e.g. ADSL, Internet. Front End Services are foreseen to manage the needs of the system and CLU. User Terminals (UTEs) and Supervisor Terminal can be connected to the system using public transmission services.

\section{Problems Concerning Tunnel Lighting}

According to Papagiannis, (2004a), tunnel lighting is one of the most challenging places where software agent-based automation can be applied. This is because lighting within tunnels follows a very complicated formula. According to the international standards of the CIE (Commission Internationale de Eclairage) (CIE 1984,
1990), the luminance L, calculated as the luminous intensity per surface unit, is not constant along the tunnel. It is different at the entrance of the tunnel, the so called threshold zone, in the transition zone, in its interior and in the exit one. It follows a curve that relates the luminance with the distance from the entrance of the tunnel and the conditions in the access zone which depend on the vehicle speed, the time of the day, the day light brightness and the design of the tunnel entrance (Papagiannis, 2004b).

In more detail the luminance level in a tunnel is divided to five zones which are:

\section{Entrance zone threshold 1}

$$
L_{t r}=k * L_{20}
$$

Where $L_{t r}$ is the lighting level in the transition zones and $L_{20}$ is the mean value of the luminance values which are encountered in a solid angle of 20 degrees with an axis from the driver's eye to a point located at $1 / 4$ of the tunnel entrance, when the vehicle is located at a distance equal to the stopping distance of the car. The coefficient $k$ is related to the tunnel entrance characteristics.

The length of the entrance zone is half of the stopping distance of a vehicle. We can see at the bottom of Figure 2 the stopping distances for various vehicle speeds.

\section{Entrance zone threshold 2}

$$
L_{t r}=L_{t h}-a * x
$$

where $L_{t h}$ is the luminance level of the previous entrance zone. This level is linearly decreasing from $100 \%$ to $40 \%$ at the end of the zone.

The length of this zone is half of the stopping distance of a vehicle.

\section{Transition zone}

$$
L_{t r}=L_{t h} *(1.9+x)^{-1.4}
$$

where $L_{t h}$ is the luminance level of the entrance zone and $x$ is the distance from the tunnel entrance.

The length of this zone is not fixed.

\section{Internal zone}

$$
\begin{array}{cl}
L_{t r}=2 \mathrm{~cd} / \mathrm{m}^{2} & \text { for the night } \\
2 \mathrm{~cd} / \mathrm{m}^{2} \leq L_{t r} \leq 15 \mathrm{~cd} / \mathrm{m}^{2} & \begin{array}{l}
\text { for the rest of } \\
\text { the day }
\end{array}
\end{array}
$$




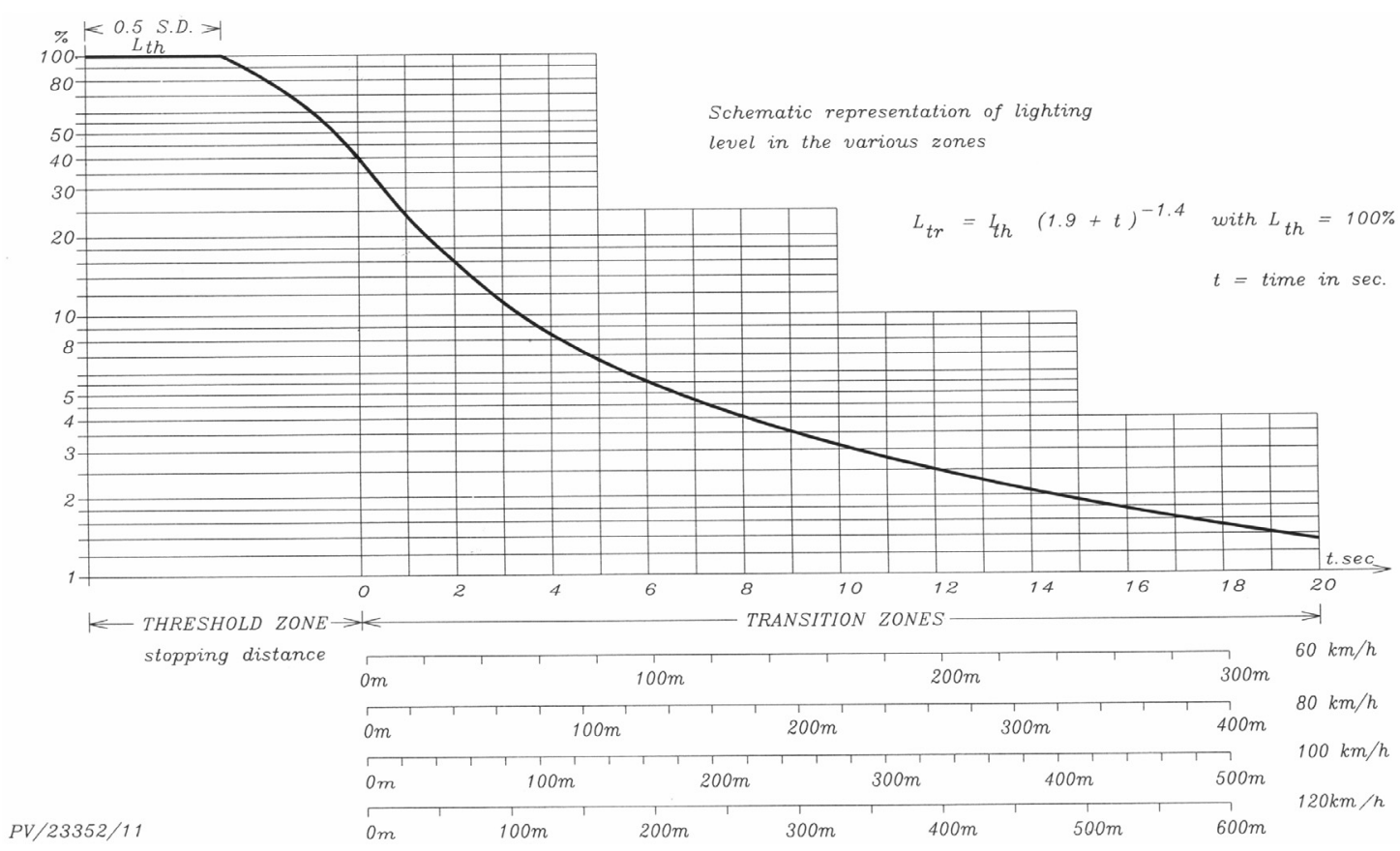

Figure 2. Light level in the various zones of a tunnel.

The luminance level of this zone is constant and depends only on the hour of the day.

The length of this zone is not fixed.

\section{Exit zone}

$$
\begin{array}{ll}
L_{t r}=2 \mathrm{~cd} / \mathrm{m}^{2} & \text { for the night } \\
L_{t r}=5 * L_{t r p} & \text { for the rest of the day }
\end{array}
$$

The luminance level of this zone is constant and depends on the hour of the day and the level of the previous zone $\left(L_{t r p}\right)$.

The length of this zone is not fixed.

In each tunnel we consider also that there is a light sensor that is connected to a specific PMS. This sensor can help the PMS calibrate the lighting system to produce the appropriate curve.

\section{System architecture}

The system was implemented using the JADE agent framework which offers a number of advantages (Moraitis, 2004). The general system architecture appears in Figure 3. As it can be noted, the system consists of the following four types of agents: PMS agent, User agent, Daylight agent, Calibration agent.

PMS agent is the normal agent that controls a specific PMS. It keeps a number of internal variables like the distance from the entrance of the tunnel, the Log agent id, the Calibration agent id and the type of lighting. It also keeps some

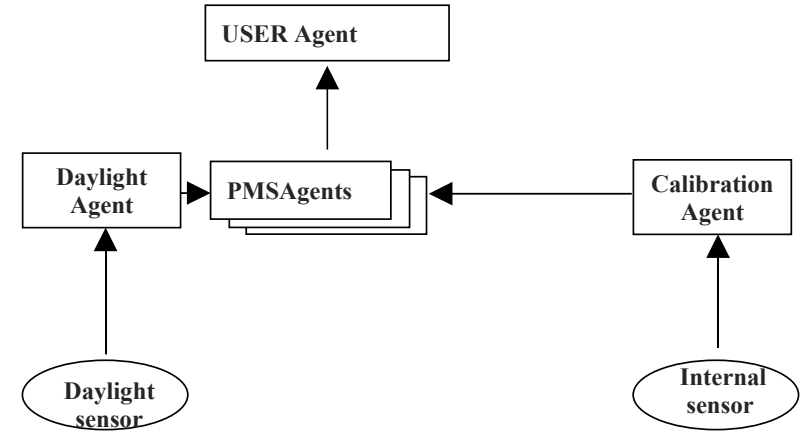

Figure 3. General system architecture.

internal variables to store necessary setting such as the current daylight level, the working hours of its lighting system, the calibration coefficient and others.

User agent is the main coordination agent that has contact with the system administrator who monitors the system. Its main purpose is to hold registration information on all agents and to keep everyone's status. Each PMS agent communicates with the log agents every time something in its state (light level, alarm, etc.) changes. It also allows the administrator to check for any problems that might be occurring with the PMS agents or the devices they control.

Daylight agent is the first agent in the tunnel and is connected with the daylight sensor outside the tunnel. It sends messages periodically to indi- 
cate change in the external light conditions. It can also be contacted by the PMS agents every time they need fresh information on the daylight conditions.

Calibration agent is internal and connected to the light sensor in the tunnel. There may be many such calibration agents if the characteristics of the tunnel are not uniform. For the sake of simplicity, in the prototype we introduced only one such agent. It sends messages periodically to indicate any change in the light conditions. It can also be contacted by the PMS agents every time they need fresh information to calibrate their lighting systems.

\section{Agent ontologies}

The semantics of information exchanged between the agents is described using agent ontologies. They provide a common understanding of the domain that is communicated across the four agents of the system. Jade provides full support of ontologies based on the FIPA standards. The three following ontologies where implemented for this system:

- Calibration ontology. All internal condition changes captured by the calibration agents are forwarded to the PMS agents using a calibration ontology which holds information concerning new calibration values.

- Daylight ontology. The daylight agent sends information using a daylight ontology which contains information regarding external conditions.

- Intensity ontology. This ontology holds intensity results calculated by the PMS agents which are sent to the User agents.

\section{Operational scenario}

Initially, when the system is started, the daylight agent sends an initial external condition reading to all available PMS agents. Also, the calibration agents communicate with the respective PMS agents to provide internal sensor readings. The PMS agents react to the daylight or calibration messages obtained by calculating new intensities and adjusting the light systems. As soon as new intensities are calculated they are also sent to the user agent for further logging. Figure 3 shows the communication among the agents. Therefore, PMS agents act autonomously as soon as they receive new messages either from the daylight agent or from their corresponding calibration agents.

Another feature would be to support collaboration among the agents themselves. This would allow them to adjust their intensities based also on readings and information offered by the two agents besides them (left and right PMS agents).

\section{Prototype development}

Based on the design described above, a prototype was implemented and a number of simulation tests were performed. As mentioned above, all agents communicate intensity results with the User agent which then logs information and outputs a graph within an HTML page to simplify evaluation. Two examples with different calibration coefficients and daylight levels can be seen in Figures 4 and 5 .

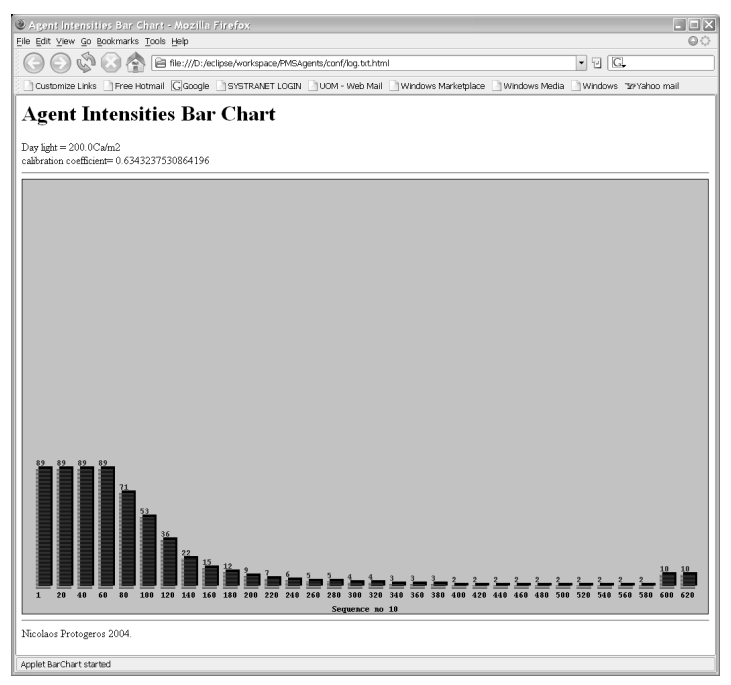

Figure 4. Daylight level $200 \mathrm{~cd} / \mathrm{m}^{2}$, calibration coefficient 0.63 .

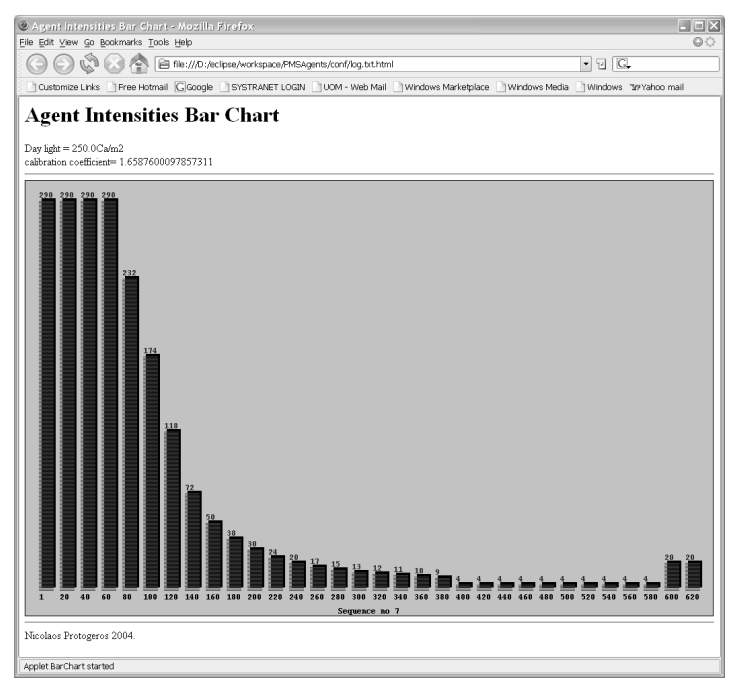

Figure 5. Daylight level $320 \mathrm{~cd} / \mathrm{m}^{2}$, calibration coefficient 0.66 . 
As it can be noted, when the daylight level is low, the agents decrease their intensity levels accordingly (Figure 4). On the other hand, when the daylight increases, so does the intensity level of all PMS agents (Figure 5). The above paradigms are two simulation runs during the day.

Jade also provides a sniffer agent that can be used to detect all message exchange among all participating agents. So, this agent produces the sequence diagram of events which simplifies debugging of collaborating messages. This can be seen in Figure 6 below.

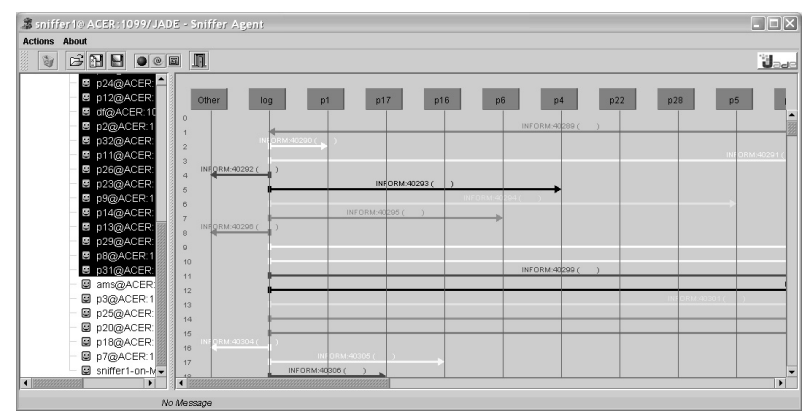

Figure 6. The sequence diagram.

\section{Conclusions}

This study presented an approach to solving the tunnel lighting problem using an agent mediated implementation. In order to satisfy the problem requirements, various agent types were developed. They exchange information regarding external lighting, calibration readings and interface data. In this approach, each agent operates autonomously to calculate optimal light intensity and adjust accordingly to continuous environmental changes.

This architecture allowed us to conclude the following issues:

- Agents can improve the efficiency of traditional tunnel lighting systems since they can adjust their light intensity independently in contrast to traditional systems that adjust groups of lights.

- The use of agents can decrease power consumption since working independently offers the possibility to decrease intensity at specific points, thus decreasing total consumption.

- Finally, depending on the operating hours of each lamp, agents can collaborate in order to balance working lamp hours. In this way, the tunnel achieves maximum availability as well as minimum maintenance and support requirements.

Future research may include further investigation of the collaboration among agents to cover possible faults of lamps. Another suggestion for future research is to investigate the case when lamps can not decrease their intensity linearly and thus there is a need to collaborate in groups in order to provide the correct light intensity for the tunnel.

\section{References}

[1] J. M. AKkermans, F. YgGe, Smart software as customer assistant in large-scale distributed load management. In Proceedings of Distribution Automation/ Demand Side Management (DA/DSM) '97., PenWell Conferences and Exhibitions, (October 1997), (Available from: http://www . enersearch.se/ygge/).

[2] J. M. Akkermans, F. Ygge, R. Gustavsson, HOMEBOTS: Intelligent decentralized services for energy management. Knowledge Management: Organization, Competence and Methodology, J. F. Schreinemakers (Ed. ), (1996), Ergon Verlag, Wuerzburg, Germany.

[3] M. Chmiel, M. Gawinecki, P. KaczmareK, M. SZYMCZAK, M. PAPRZYCKI, Efficiency of JADE Agent Platform. Scientific Programming, JADE Workshop at AAMAS, (2005).

[4] CIE, Road surfaces and lighting. Joint technical report CIE/PIARC, pp. 66-1984.

[5] CIE, Guide for the lighting of road tunnels and underpasses. pp. 88-1990.

[6] R. A. Gagliano, M. D. Fraser, M. E. Schaefer, Auction Allocation of Computing Resources. Communications of the ACM, Vol. 38, No. 6, (June 1995), pp. 88-102.

[7] R. Gustavsson, Agents with Power. Communications of the ACM, (March 1999), Vol. 42, No. 3.

[8] JADE, http://jade.tilab.com/

[9] J. F. Kurose, R. Simha, A Microeconomic Approach to Optimal Resource Allocation in Distributed Computer Systems. IEEE Transactions on Computers, Vol. 38, No. 5, (May 1989), pp. 705717.

[10] J. H. J. Lenting, P. J. BRASPENNING, Delegated Negotiation for Resource Reallocation. Lecture Notes in Artificial Intelligence, Vol. 671, pp. 299-312, (1993), Springer Verlag, Berlin. 
[11] P. Moraitis, N. Spanoudakis, Combining Gaia and JADE for Multi-Agent Systems Development. 4th International Symposium "From Agent Theory to Agent Implementation" (AT2AI4), in:Proceedings of the 17th European Meeting on Cybernetics and Systems Research (EMCSR 2004), (April 2004), Vienna, Austria.

[12] B. Nilsson, P. Dokopoulos, G. Papagiannis, D. TAMPAKIS, N. LETTAS, G. BIANCONNI, G. FABOZZI, A PLC-Based Electrical Energy Consumption Management System - Preliminary Field Test Results. Proceedings of the 7th International Symposium on PLC, (2003), Kyoto, Japan, pp. 10-15.

[13] G. Papagiannis, K. Gouramanis, T. PAPADOPOUlos, K. PAPADOPOUlOS, P. DOKOPOUlos, A PLC Based Energy Consumption Management System - Performance Analysis of Overhead Lines and Underground Cables: Measurements and Simulation. Proceedings of the MedPower04 Conference, (November 2004), Lemesos, Cyprus.

[14] G. Papagiannis, D. Tsiamitros, K. Gouramanis, G. ANDREOU, P. DoKopoulos, A PLC Based Energy Consumption Management System - Pilot Installation Field Tests and Simulation Results. Proceedings of the 8th International Symposium on PLC, (March 30-April 2 2004), Zaragoza, Spain.

[15] PMS: PROJECT ACRONYM POWERMAN SWITCH, EU Programme Acronym: EESD. Project Reference: NNE5/247/2001,

URL: http://www.pmsengineering.com/.

[16] D. Tsiamitros, N. Lettas, G. Papagiannis, D. TAMPAKIS, A PLC-based Energy Consumption Management System - Field tests and Simulation results. Proceedings of the 38th Universities Power Engineering Conference (UPEC), (September 2003), Thessaloniki, Greece.

[17] F. YGGE, Market-Oriented Programming and its Application To Power Load Management. PhD thesis, Department of Computer Science, Lund University, (1998). ISBN 91-628-3055-4, CODEN LUNFD6/(NFCS-1012)/1-224/(1998) (Available from http: //www. enersearch.se/ygge/).

[18] F. YGGE, Energy Resellers-An Endangered Species? EnerSearch Public Report: EnS, 99:02, v2.0/990525, (1999).

[19] F. YgGe, R. Gustavsson, H. AKKeRMans, HOMEBOTS: Intelligent Agents for Decentralized Load Management. Proceedings of DA/DSM 96 Europe Distribution Automation \& Demand Side Management, (October 1996), Vienna, Austria.

[20] F. YgGe, J. M. AKkeRmans, Power load management as a computational market. Proceedings of the Second International Conference on Multi-Agent Systems, Kyoto, Japan, (December 1996), AAAI Press, Menlo Park, California.

[21] F. YgGe, J. M. AKKeRMans, On resource-oriented multi-commodity market computations. In Proceedings of the Third International Conference on MultiAgent Systems, Paris, (July 1998), IEEE Computer Society, Los Alamitos, California.
Received: February, 2006 Revised: February, 2007

Accepted: February, 2007

Contact addresses:

Nicolaos Protogeros

University of Macedonia 156 Egnatia Street

GR - 54006 Thessaloniki, Greece

Anastasios A. Economides University of Macedonia 156 Egnatia Street GR - 54006 Thessaloniki, Greece e-mail: economid@uom.gr

Grigoris K. Papagiannis Dept. of Electrical and Computer Eng. Aristotle University of Thessaloniki GR - 54124 Thessaloniki, Greece

Chris Syleos

Dept. of Electrical and Computer Eng. Aristotle University of Thessaloniki GR - 54124 Thessaloniki, Greece

NicolaOs PROTOGEROS is a lecturer of Electronic Commerce at the University of Macedonia, at Thessaloniki, Greece. He holds a Ph.D. in information technology from National Polytechnique Institute in France, a DEA on image processing from the University of Paul Sabatier, Toulouse, France, and a B.S. in mathematics from Aristotle University of Thessaloniki. He has worked in the Information Technology sector for many years specializing in Electronic Commerce applications. He has been a project leader in many research and development projects in the area of Web-based technologies, Software Agents and Virtual Enterprises. He has published articles on Electronic Commerce, Information Technologies and Network Management.

ANASTASIOS A. ECONOMIDES received the Diploma degree in Electrical Engineering from Aristotle University of Thessaloniki, in 1984. Holding a Fulbright and a Greek State fellowships, he received a M.Sc. and a Ph.D. degree in Computer Engineering from the University of Southern California, Los Angeles, in 1987 and 1990, respectively. He is the director of CONTA (Computer Networks and Telematics Applications) laboratory at the University of Macedonia, Thessaloniki, Greece. He has published over 120 peer-reviewed papers.

GRIGORIS K. PAPAGIANNIS was born in Thessaloniki, Greece, on September 23, 1956. He received his Dipl. Eng. degree and the Ph.D. degree from the Department of Electrical and Computer Engineering at Aristotle University of Thessaloniki, in 1979 and 1998 respectively. He is currently As. Professor in the Power Systems Laboratory of the Department of Electrical and Computer Engineering at Aristotle University of Thessaloniki, Greece. His special interests are power systems modelling, computation of electromagnetic transients, distributed generation and powerline communications.

CHRIS SylEOS received a BSc degree in Computer Science from the University of Sussex, England in 1993, and MSc degree in Parallel and Distributed Systems from the University of Westminster, England in 1995. Currently he is a PhD candidate in the Department of Electrical and Computer Engineering at Aristotle University of Thessaloniki. His research interests are in the areas of intelligent agents and e-commerce related with the restructuring of the electric utility industry. 